\title{
Regulatory roles of microtubule- associated proteins in neuronal morphogenesis. Involvement of the extracellular matrix
}

\section{G. Ramírez ${ }^{1}, A$. Alvarez ${ }^{1}$ \\ J. Garcia-Abreu², \\ F.C.A. Gomes', \\ V. Moura-N eto ${ }^{2}$ and \\ R.B. Maccioni ${ }^{1}$}

\author{
'Laboratorio de Biología Celular y Molecular, Facultad de Ciencias, \\ Universidad de Chile, Santiago, Chile \\ ${ }^{2}$ Laboratório de Morfogênese Celular, Departamento de Anatomia, \\ Universidade Federal do Rio de Janeiro, Rio de Janeiro, RJ, Brasil
}

\section{Correspondence \\ R.B. Maccioni \\ Laboratorio de Biología Celular \\ y Molecular \\ Facultad de Ciencias \\ Universidad de Chile \\ Las Palmeras 3425, Ñ uñoa \\ Santiago \\ Chile \\ Fax: + 56-2-218-6245 \\ E-mail: rmaccion@ abello.dic.uchile.cl \\ Presented at the 5th Brazilian Symposium on Extracellular Matrix - SIM EC, Angra dos Reis, RJ, Brasil, September 7-10, 1998. \\ Research partially supported by the Presidential Chair on Science (Chile), and by an international CNPq-Conicyt cooperation project.}

Received November 17, 1998 Accepted December 21, 1998

\section{Abstract}

As a result of recent investigations, the cytoskeleton can be viewed as a cytoplasmic system of interconnected filaments with three major integrative levels: self-assembling macromolecules, filamentous polymers, e.g., microtubules, intermediate filaments and actin filaments, and supramolecular structures formed by bundles of these filaments or networks resulting from cross-bridges between these major cytoskeletal polymers. The organization of this biological structure appears to be sensitive to fine spatially and temporally dependent regulatory signals. In differentiating neurons, regulation of cytoskeleton organization is particularly relevant, and the microtubule-associated protein (MAP) tau appears to play roles in the extension of large neuritic processes and axons as well as in the stabilization of microtubular polymers along these processes. Within this context, tau is directly involved in defining neuronal polarity as well as in the generation of neuronal growth cones. There is increasing evidence that elements of the extracellular matrix contribute to the control of cytoskeleton organization in differentiating neurons, and that these regulations could be mediated by changes in MAP activity. In this brief review, we discuss the possible roles of tau in mediating the effects of extracellular matrix components on the internal cytoskeletal arrays and its organization in growing neurons.

\section{Introduction}

The molecular factors that affect neuronal morphogenesis and its differentiation constitute a central paradigm in modern cell biology. The structure and integrity of the cytoplasm of eukaryotic cells depend on the assembly and organization of the network of interlinked filaments that form the cytoskel-

\section{Key words}

- Extracellular matrix

- Tau functions

- Regulatory patterns

- Neuronal morphogenesis eton: microtubules, actin filament and intermediate filaments (1). These polymers, which exhibit variable degrees of assembly dynamics (2), coexist as a result of multiple macromolecular interactions that determine the precise intracellular distribution of cytoskeletal filaments, covering wide domains within the cytoplasm, and interacting with most of the cellular organelles, including mitochondria, 
centrosomes, lysosomes, and the cell nucleus and membrane.

The structural and functional organization of living cells, and therefore the spatial array of their components, are generated through a process of growing complexity. Cell morphology is determinant for specific cell functions, and therefore the architecture of cells appears to be finely regulated in response to structural demands of cells within the context of their functions. The cytoskeleton is a system of interconnected filaments, in which three major integrative levels can be distinguished: self-assembling macromolecules, filamentous polymers, e.g. microtubules, intermediate filaments and actin filaments, and supramolecular structures formed by bundles of these filaments or cross-bridges between these major cytoskeletal polymers (Figure 1). When cytoplasmic macromolecules assemble into supramolecular structures that shape the cytoskeleton, a set of properties of living cells emerge, such as their capacity of dividing, growing, moving and changing their morphology during differentiation. The architecture of cells is provided to a large extent by the cytoskeletal organization (3). However, in addition to the internal components and regulatory signals, extracellular events can also contribute to the complex regulatory patterns of cytoskeletal organization (4).

In the analysis of the different associations between cytoskeletal components, a major question emerges: how can cytoplasmic macromolecules or extracellular signals contribute to cytoplasmic organization and neuronal shape? On the other hand, how is this structure modulated as a consequence of the multiple functional demands of neuronal cells? The study of protein-to-protein interactions that play a key role in the structure of the cytoskeletal network has been one of the main aspects of our recent investigations (5).
Figure 1 - Simplified scheme showing the three integrative levels in the process by which macromolecular units assemble into cytoskeletal polymers, and their association to form supramolecular structures, mainly bundles of these polymers, and networks involving microtubules (MT), intermediate filaments (IF) (neurofilaments) and actin filaments (AF). Microtubule-associated proteins (MAPs) appear to play a major modulatory role at the level of microtubule assembly and in regulating the linkages between the different filaments of the cytoskeleton. T, Tubulin; $A$, actin; ABPs, actin binding proteins; IFPs, intermediate-filaments proteins.

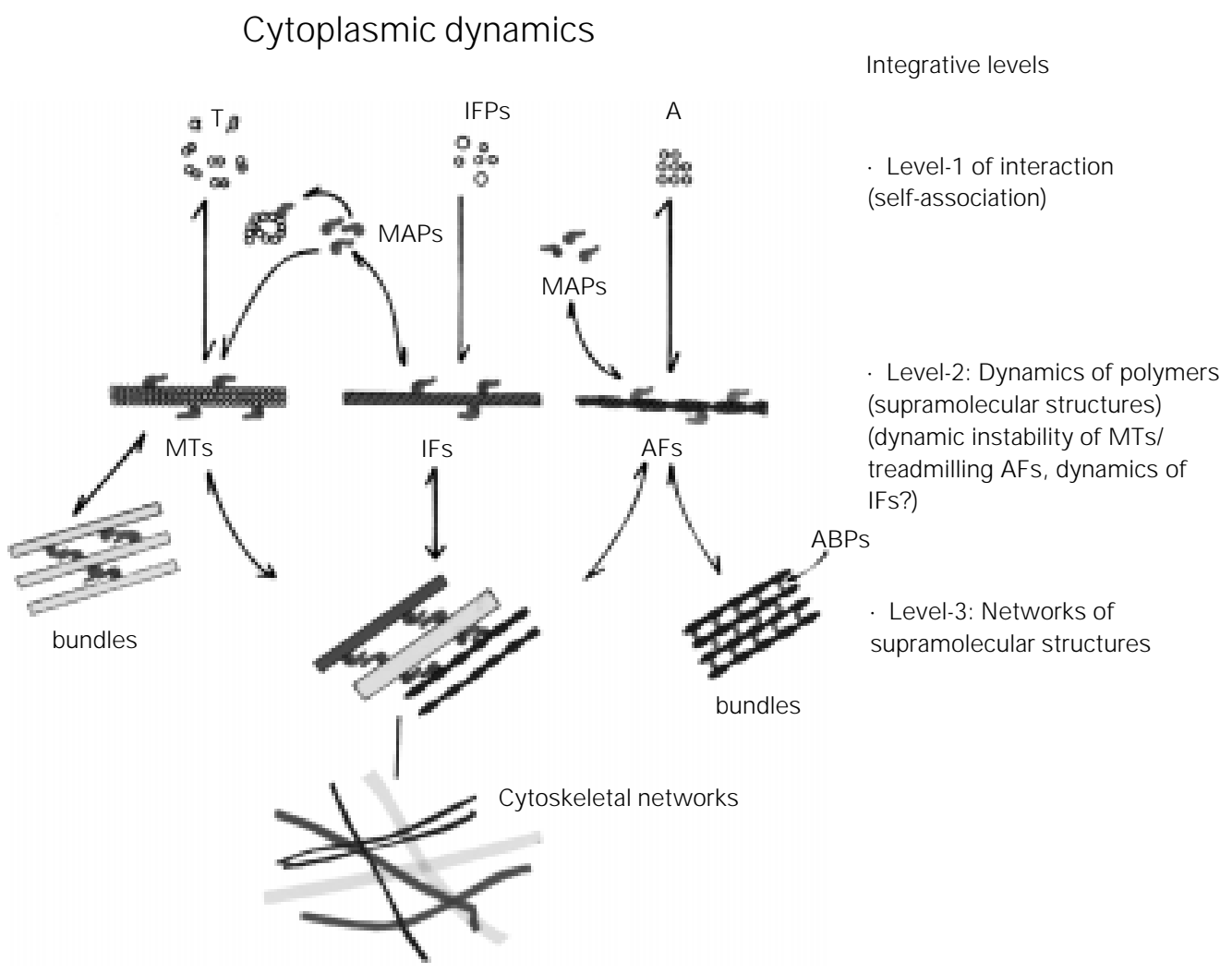


These interactions include: i) homologous associations like those occurring in the oligomerization of actin in the assembly of microfilaments or self-assembly of tubulin leading to microtubule formation, and ii) heterologous associations like the interactions between cytoskeletal polymers and microtubule-associated proteins (MAPs). However, there is another branch in this field of study, which is the involvement of the extracellular matrix (ECM) in the determination of modulatory signals that affect the neuronal cytoskeleton in processes like neuritogenesis, and the possible role of MAPs in mediating these cellular changes. This is the main focus of the present analysis.

\section{Factors influencing microtubule organization and neurite stability and outgrowth}

Neuronal microtubules constitute major structures directly involved in neuronal morphogenesis, with different subsets of tubulin specifically expressed in neuronal and glial cells (6). Their assembly and dynamics appear to be modulated by MAPs. Different animal tissues contain variable sets of MAPs, and the cell type-specificity of these proteins may account for their differential expression in each type of cell (7). In experiments in which cDNAs of tau and MAP-1B were transfected into HeLa cells and fibroblasts, microtubules were stabilized against depolymerizing agents (8). Post-translational modifications of microtubules are directly involved in the determination of morphogenesis in neurons (9). The fact that these microtubules are enriched in acetylated tubulin suggests that this post-translational modification of tubulin renders microtubules more resistant to depolymerization. In N2A neuroblastoma cells, studies using depolymerizing agents demonstrated that tau colocalized in a discrete fashion along stable microtubular polymers, while a more continuous association of tau along less stable microtubules containing tyrosinated tubulin was also observed (10). The experiments indicate that there is a differential association of tau isoforms into stable and weak microtubules, and that tau association into more dynamic polymers containing tyrosinated tubulin contributes to their stabilization.

Further evidence of the roles of MAPs in neuritogenesis comes from studies on N18 neuroblastoma cells, which grow as loosely attached and morphologically undifferentiated spherical cells. However, removal of serum leads to greater adherence to a substratum and to the initiation of neurite outgrowth (11). Neurite differentiation begins within minutes and differentiated morphology is attained within 3 days in the low-serum environment. Even though the nature of extracellular matrix components used as substrate contributes to neuronal outgrowth, initiation of neuritogenesis is not simply a matter of adhesion, since $\mathrm{N}-18$ cells grown over poly-D-lysine but in the presence of $10 \%$ serum do not extend neurites. Studies have indicated a role of MAPs in regulating microtubule assembly during nerve fiber outgrowth (12). High speed supernatants from $\mathrm{N}-18$ differentiated cells were active in inducing in vitro microtubule assembly from purified brain tubulin, but extracts of undifferentiated cells were unable to promote microtubule polymerization.

\section{The role of tau in neuronal growth}

An increasing amount of information supports the role of neuronal tau proteins in the outgrowth of neuronal processes and the establishment of neuronal polarity $(13,14)$. Tau induces tubulin assembly into microtubules, stabilizes these cellular polymers, and controls their dynamic instability by affecting the rates of polymerization and transition to catastrophic depolymerization (15). Thus, an integrated view of the neuronal form of tau led to the suggestion that its major role is 
to regulate microtubule assembly and dynamics. This is supported by a substantial increase in tau expression concomitant with neurite extension (16). In essence, the changes in the expression of tau in transfected cells appear to play a critical role in the ability of tau to promote neuritic stability in cultured cells (17).

Early microinjection studies have pointed out that the in vivo actions of neuronal tau to induce and stabilize microtubules, which are necessary for neuronal activity, may not be sufficient to explain neuronal morphogenesis (18). Therefore, an important role revealed for tau is the directing of the neuronal outgrowth process from the cell soma to the distal ends of axon-like neurites (19). Within this context it is proposed that critical targets for the action of tau on neuronal growth are precisely the distal axons and growth cones (19). In fact, in polarized hippocampal neurons, the enzyme cdk-5 becomes concentrated at the distal tip of growing axons, associating with the subcortical cytoskeleton (20). Also, it was shown that specific phosphorylations of MAP-1b occur during neuronal outgrowth in neuroblastoma cells, and this modification is related to an enhancement of microtubule assembly (21). Therefore, besides tau the high-molecular weight MAPs (MAP-1b, MAP-3) also appear to be involved in neuronal growth (22).

A major question to address is how tau function is regulated. At this level it is worth mentioning that tau proteins are produced by a single gene by alternative splicing, which yields several protein products. Thus, splicing of a common RNA transcript, as well as post-translational modification of the tau molecule are the main regulatory mechanisms for tau activity on neurons. The single tau gene contains 14 exons encoding for the six different isoforms of this protein (for a review, see 5). Expression of tau isoforms is developmentally regulated, with some differences in the central and peripheral nervous system. In the fetal brain, only the short tau isoforms containing only three repetitive binding motifs are expressed, but after postnatal development neuronal cells can express all six major isotypes of tau. A marked decrease in fetal tau expression occurs in rats after postnatal day 8 . At the level of the peripheral nervous system, only the highmolecular weight $(110 \mathrm{kDa})$ isoform of tau containing two additional exons is expressed (23).

Within this context, another aspect to emphasize in the analysis of cellular factors affecting the extension of neurites by neuronal cells is the role of tau in mediating microtubule-actin filament interactions $(5,10,24)$, an activity that could be involved in regulating the actin cortex and association with plasma membrane in the establishment of architectural patterns during neuronal growth (25).

\section{Extracellular matrix cytoskeleton and MAPs in the context of neuronal growth}

Recent reports have raised the possibility of the involvement of tau as an integral part of the molecular machinery that controls the establishment of neuronal polarity, and have indicated that this protein modulates the expression of extracellular signals and their consequence for neuronal architecture during axonal growth. The main question is how elements of the extracellular matrix become translated into changes in cell morphology and activity. Even though most of the reported data point to tau in the intraneuronal context, recent observations have shed light on the extracellular events involved in neuronal growth. Laminin, a major extracellular matrix molecule, is capable of stimulating the extension of axonal processes as related to MAP-1b phosphorylation (26). On the basis of redundancy in MAP-1b and tau functions (26), and further observations on the behavior of neurons from transgenic animals (27), it is reasonable to think that tau, 
which is a neuronal substrate of cdk-5, is involved in mediating the laminin effects on neuronal growth. Previous observations have suggested that cdk-5 may serve as a regulatory linker between environmental signals of ECM elements, and MAPs involved in the axonal formation process (26). The effects of laminin appear to be linked to specific phosphorylations of either MAP-1b or tau, and a reorganization of these MAPs within the neuronal cytoskeleton. These findings are strongly supported by additional observations that inhibition of certain protein kinases abolishes the ECM-mediated promotion of neuronal polarity (28). A schematic representation of the effects of the ECM is illustrated in Figure 2.

Extracellular matrix components appear to affect the direction of neurite outgrowth by interacting with the growth cone; how- ever, the way they function has not been elucidated. Elements of the ECM, such as laminin, stimulate neuronal growth (29), but are also involved in modulating cell contacts and the spreading phenomenon (30). The concept of a differential cell adhesion provided by ECM elements is restricted to substrate conditions, and the molecular nature of these elements (4). When growth cones are experimentally detached, they show a greater level of contact with collagen and fibronectin substrates as compared with laminin. However, there is evidence that laminin is a significantly better substrate than fibronectin and collagen (31). Evidence against differential adhesion as a mechanism for laminin function is that adhesion is not a determinant for growth cone preference for substrates. Outgrowth of neurons in culture is faster on a laminin substrate than on a

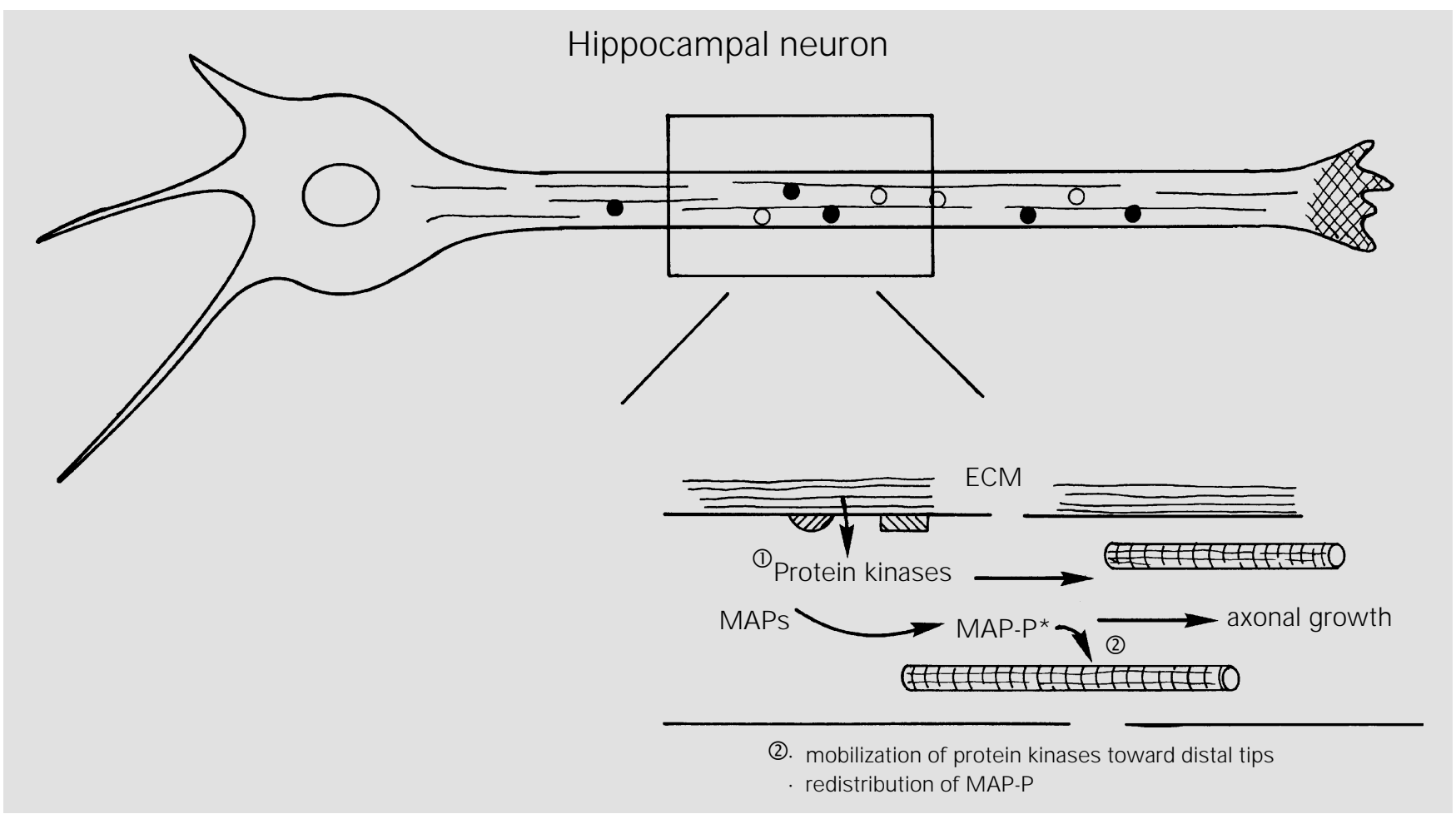

Figure 2 - Schematic representation of a hippocampal neuron, and the effects of the extracellular matrix (ECM) in stimulating axonal extension in growing neurons. The scheme indicates that ECM effects (laminin, others) can be transduced in a sequence of molecular events (1 and 2 ) leading to phosphorylation of neuronal MAPs and incorporation of MAP-P (phosphorylated MAPs) into growing microtubules, and/or activation and redistribution of protein kinases that could be critical for neuronal growth. In the upper part of the figure, closed circles denote differentially phosphorylated MAPs at specific sites, and open circles the normally phosphorylated MAPs. 
control substrate, but eventually neurons achieve polarity on either substrate (32). On the other hand, besides laminin, neuronal proteoglycans are directly involved in the outgrowth of fetal hippocampal neurons $(33,34)$. However, the precise mechanisms by which tau protein and other MAPs directly involved in neuronal polarity participate in the action of ECM components are not clear at all.

\section{Extracellular matrix and the glial cytoskeleton}

Elements of the ECM are also known to

Table 1 - Morphometric analysis of hippocampal cells.

${ }^{1}$ Hippocampal cells in primary cultures were plated onto culture dishes and incubated under substrate control conditions, or in the presence of either ECM element. LN, Laminin, HS, heparan sulfate.

\begin{tabular}{lcccc}
\hline & \multicolumn{4}{c}{ Neuritogenesis of embryonic rat hippocampal cells } \\
\cline { 2 - 5 } & Control ${ }^{1}$ & Laminin & Heparan-sulfate & LN + HS \\
\hline Number of cells/field & 296 & 308 & 207 & 254 \\
$\begin{array}{l}\text { \% Cells bearing processes } \\
\text { Fraction of processes }\end{array}$ & $21 \%$ & $27 \%$ & $14 \%$ & $36 \%$ \\
$\begin{array}{l}\text { that are axon-like (of total } \\
\text { cells bearing processes) }\end{array}$ & 0.36 & 0.41 & 0.22 & 0.49 \\
Average length of processes $(\mu \mathrm{m})$ & $21 \pm 10$ & $32 \pm 14$ & $17 \pm 9$ & $43 \pm 19$
\end{tabular}

Table 2 - In vitro polymerization of tubulin in the presence of brain extracts of primary cultures of hippocampal cells.

${ }^{1}$ Net assembly obtained after subtraction of the value of control for tubulin selfaggregation in the absence of any cell extract. Final concentration of proteins in the assembly system: $1.5 \mathrm{mg} / \mathrm{ml}$ tubulin; $0.14 \mathrm{mg} / \mathrm{ml}$ brain extracts. ${ }^{2} \mathrm{Hippocampal}$ cells in primary cultures were incubated under substrate control conditions, or in the presence of either ECM element. LN, Laminin; HS, heparan sulfate. Tubulin assembly was monitored by the sedimentation assay (40).

Net assembly ${ }^{1}$ ( $\mu \mathrm{g}$ microtubules)
Tubulin control (no factors added)

Tubulin + brain tau

Tubulin + control brain extract ${ }^{2}$

Tubulin + extract incubated with LN

Tubulin + extract incubated with HS

Tubulin + extract incubated with LN + HS
0.0

$49.9 \pm 5.1$

$10.4 \pm 2.2$

$16.4 \pm 4.6$

$14.8 \pm 3.0$

$34.5 \pm 2.7$ affect the cytoskeleton and morphological changes in cultured astrocytes (35). It was reported that transforming growth factor (TGF)-beta1 stimulates the production of laminin and fibronectin and their incorporation into the ECM of astrocytes. Furthermore, it is possible that a TGF-beta1-like growth factor is secreted by neuronal cells and induces the glial fibrillary acidic protein (GFAP) gene (36). This factor also promoted formation of stress fibers and increased the content of actin. These changes in cell shape, cytoskeleton and ECM promoted by TGF beta 1 may be of relevance for understanding the connections between ECM and cell cytoskeleton in the context of brain development (37). On the other hand, T3 hormone and conditioned media from cerebellar T3-treated astrocytes enriched with EGF and TNF-beta (36) promoted alterations in the GFAP cytoskeleton (38) and fibronectin. Fibronectin, which usually exhibits a discontinuous distribution of control, on the cell surface became diffuse in T3-treated astrocytes (39). Furthermore, this conditioned medium has been recently reported to induce cerebellar neuronal proliferation due to the presence of EGF and TNF-beta.

\section{Tau proteins could be sensitive to the modulatory effects of ECM on neuronal outgrowth}

An interesting observation comes from studies indicating that subsets of tau may act differentially in tau interactions with microtubules, actin and neurofilaments (24). It is also possible that certain isoforms of tau may respond with different sensitivity to the effects of ECM components on neuritogenesis. However, there is no clear evidence of the specific roles of tau isoforms due to limitations in generating probes to examine their specific roles. Tau function appears to be modulated by ECM and this regulation seems to depend on the nature of ECM elements. In a study of neurite extension in 
hippocampal cells in primary cultures, laminin stimulated the number of neurons bearing processes, an effect potentiated by the presence of heparan sulfate. An increase was also observed in the fraction of processes that are axon-like as well as in the average length of processes; however, heparan sulfate by itself failed to produce this effect (Table 1). Therefore, an experiment was carried out in which tubulin polymerization was analyzed in the presence of hippocampal brain extracts obtained from cells cultured under different ECM experimental conditions. Cell that were incubated with laminin and heparan sulfate showed the highest activity of factors inducing microtubule assembly as compared with controls incu- bated with purified brain tau (Table 2). Cosedimentation studies revealed that a higher fraction of tau was associated with polymers obtained from pure tubulin and extracts of the laminin-heparan sulfatetreated cells. These studies shed further light on the molecular analysis of the roles of MAPs, and particularly tau proteins, in the sequence of events by which ECM affects neuritogenesis.

\section{Acknowledgments}

We thank Dr. Alfredo Cáceres, Lorena Saragoni and Rodrigo Toro for helpful discussions.

\section{References}

1. Porter K (1984). The cytomatrix: a short story of its study. J ournal of Cell Biology, 99 (Suppl): 3s-12s.

2. Mitchison T (1992). Compare and contrast actin filaments and microtubules. Molecular Biology of the Cell, 3: 13091315.

3. Maccioni RB \& Arechaga J (1987). The Cytoskeleton in Cell Differentiation and Development. IRL Press, Oxford.

4. Luckenbill-Edds L (1997). Laminin and the mechanism of neuronal growth. Brain Research Reviews, 23: 1-27.

5. Maccioni RB \& Cambiazo V (1995). Role of microtubule-associated proteins in the control of microtubule assembly. Physiological Reviews, 75: 835-863.

6. Moura Neto $V$, Mallat $M$, J eantet $C \&$ Prochiantz A (1986). Microheterogeneity of tubulin proteins in neuronal and glial cells from the mouse brains in culture. EMBO J ournal, 2: 1241-1248.

7. Maccioni (1986). Molecular Cytology of Microtubules. Basque Country University Press-Springer-Verlag, Leioa.

8. Takemura R, Okabe S, Umeyama T, Kanai Y, Cowan NJ \& Hirokawa N (1992). Increased microtubule stability and alpha tubulin acetylation in cells transfected with microtubule-associated proteins MAP1B, MAP-2 or tau. J ournal of Cell Science, 103: 445-456.

9. Bulinski J C \& Gundersen GG (1991). Stabilization of post-translational modification of microtubules during cellular morphogenesis. Bioessays, 13: 285-293.

10. Maccioni RB, Tapia $L \&$ Cambiazo V (1995). Functional organization of tau proteins during neuronal differentiation and development. Brazilian J ournal of Medical and Biological Research, 28: 827-841.

11. Seeds NW \& Maccioni RB (1978). Proteins from morphologically differentiated neuroblastoma cells promote tubulin polymerization. J ournal of Cell Biology, 78: 547-555.

12. Seeds NW \& Maccioni RB (1987). Characterization of a microtubule-assembly promoting factor in differentiated neuroblastoma cells. In: Maccioni RB \& Arechaga J (Editors), The Cytoskeleton in Cell Differentiation and Development. IRL Press, Oxford, 187-192.

13. Cáceres A \& Kosik K (1990). Inhibition of neurite polarity by tau anti-sense oligonucleotides in primary cerebellar neurons. Nature, 343: 461-463.

14. Gordon-Weeks P (1991). Growth cones: the mechanism of neurite advance. Bioessays, 13: 235-239.

15. Trinczek B, Biernat J, Bauman K, Mandelkow EM \& Mandelkow E (1995). Domains of tau protein, differential phosphorylation, and dynamic instability of microtubules. Molecular Biology of the Cell, 6: 1887-1902.

16. Ferreira A, Busciglio J \& Cáceres A (1989). Microtubule formation and neurite growth in cerebellar macroneurons which develop in vitro: evidence for the involvement of the microtubule associated proteins MAP-1a, MAP2 and tau. Developmental Brain Research, 49: 215-228.

17. Esmaeli-Azad B, McCarty J H \& Feinstein SC (1994). Sense and antisense transfection analysis of tau function: tau influences net microtubule assembly, neurite outgrowth and neuritic stability. J ournal of Cell Science, 107: 869-879.

18. Drubin D \& Kirschner MW (1986). Tau protein function in living cells. J ournal of Cell Biology, 103: 2739-2745.

19. Black MM, Slaughter T, Moschiach S, Obrocka M \& Fisher I (1996). Tau is enriched on dynamic microtubules in the distal region of growing axons. J ournal of Neuroscience, 16: 3601-3619.

20. Pigino G, Paglini G, Ulloa L, Avila J \& Cáceres A (1997). Analysis of the expression, distribution and function of cyclin dependent kinase cdk-5 in developing cerebellar macroneurons. J ournal of Cell Science, 110: 257-270.

21. Diaz-Nido J , Serrano L, Méndez E \& Avila J (1988). A casein kinase II-related activity is involved in phosphorylation of microtubule-associated protein MAP-1b during neuroblastoma cell differentiation. J ournal of Cell Biology, 106: 2057-2065.

22. Matus A (1991). Microtubule-associated proteins and neuronal morphogenesis. J ournal of Cell Science, 15 (Suppl): 61-67. 
23. Couchie D, Mavilia C, Georgieff IS, Liem RK \& NunezJ (1992). Primary structure of high-molecular weight tau present in the peripheral nervous system. Proceedings of the National Academy of Sciences, USA, 89: 4378-4381.

24. Henriquez J P, Vial $C \& \&$ Maccioni RB (1995). Subpopulations of tau interacts with microtubules and actin filaments in different cell types. Cell Biochemistry and Function, 13: 239-250.

25. Brandt R (1996). The tau proteins in neuronal growth and development. Frontiers in Biosciences, 1: 118-130.

26. DiTella M, Feiguin F, Carri N \& Cáceres A (1996). MAP-1b/tau functional redundancy during laminin-enhanced axonal growth. J ournal of Cell Science, 109: 467-477.

27. Harada A, Ogushi K, Okabe S, Kuno J, Terada S, Oshima T, Sao Y, Takei T \& Hirokawa N (1994). Altered microtubule organization in small caliber axons of mice lacking tau protein. Nature, 369: 488-491.

28. Lochter A \& Schachner M (1997). Inhibitors of protein kinases abolish ECM-mediated promotion of neuronal polarity. Experimental Cell Research, 235: 124-129.

29. Garcia-Abreu J, Cavalcante LA \& Moura Neto V (1995). Differential patterns of laminin expression in lateral and medial midbrain glia. NeuroReport, 6: 761-764.

30. M ooney DJ , Langer R \& Ingber DE (1995). Cytoskeletal filament assembly and the control of cell spreading and function by extracellular matrix. J ournal of Cell Science, 108: 2311-2320.

31. Gundersen RW (1988). Interference reflection microscopic study of dorsal root growth cones on different substrates: assessment of growth cone-substrate contacts. J ournal of Neuroscience Research, 21: 298-306.

32. Lochter A, Taylor J, Fuss B \& Schachner $M$ (1994). The extracellular matrix molecule janusin regulates neuronal morphology in a substrate and culture time-dependent manner. European J ournal of Neuroscience, 6: 597-606.

33. Wang W \& Dow KE (1997). Effects of neuronal proteoglycans on activity-dependent growth responses of fetal hippocampal neurons. Molecular Brain Research, 48: 355-366.

34. Fernaud I, Nieto-Sampedro $M \&$ Bovolenta P (1994). Differential effects of glycosaminoglycans on neurite outgrowth from hippocampal and thalamic neurons. J ournal of Cell Science, 107: 1437-1448.

35. Matthiessen HP, Schmalenbach $C \&$ Muller HW (1989). Astroglial-released neurite growth inducing activity for embryonic hippocampal neurons is associated with laminin bound in sulfated complex and free fibronectin. Glia, 2: 177-188.

36. Gomes FCA, Maia CG, Menezes JRL \& Moura Neto V (1999). Cerebellar astrocytes treated by thyroid hormone modulate neuronal proliferation. Glia (in press).

37. Baghdassarian D, Toru-Delbauffe D, Gavaret J \& Piere M (1993). Effects of transforming growth factor beta 1 on the ECM and cytoskeleton of cultured astrocytes. Glia, 7: 193-202.

38. Lima FRS, Trentin A, Rosenthal D, Chagas C \& Moura Neto V (1997). Thyroid hormone induces protein secretion and morphological changes in astroglial cells with an increase in expression of glial fibrillary acidic protein. J oumal of Endocrinology, 154: 167-175

39. Trentin AG \& Moura Neto V (1995). T3 affects cerebellar astrocyte proliferation, GFAP and fibronectin organization. NeuroReport, 6: 293-296.

40. Cross D, Dominguez J, Maccioni RB \& AvilaJ (1991). MAP-1 and MAP-2 binding sites at the C-terminus of beta-tubulin. Biochemistry, 30: 4362-4366. 